\title{
Human bone marrow-derived mesenchymal stem cells from different bone sources: a panorama
}

\author{
Angelo V. Vasiliadis ${ }^{1,2}$, Nikiforos Galanis ${ }^{2}$ \\ ${ }^{1} 2^{\text {nd }}$ Orthopaedic Department, General Hospital of Thessaloniki "Papageorgiou”, Ring Road-N. Eukarpia, 56403 Thessaloniki, Greece; ${ }^{2}$ School of \\ Medicine, Aristotle University of Thessaloniki, University Campus, 54124 Thessaloniki, Greece \\ Correspondence to: Angelo V. Vasiliadis, MD, MSc, PhD. 2nd Orthopaedic Department, General Hospital of Thessaloniki "Papageorgiou”, 56403 \\ Thessaloniki, Greece. Email: vasiliadis.av@gmail.com.
}

\begin{abstract}
Regenerative medicine is a promising field in orthopaedic surgery. Although surgical treatments can produce excellent outcomes and may be the best choice for some patients, regenerative medicine can provide with more minimally-invasive treatment options. Mesenchymal stem cells (MSCs) are multipotent cells and are highly capable to differentiate into osteocytes or chondrocytes, while they can be isolated from different bone sources. The bone marrow aspiration from the posterior iliac crest appears to be preferred, as it provided a modestly higher concentration of nucleated cells $\left[(25.1-54.7) \times 10^{6}\right.$ cells $\left./ \mathrm{mL}\right]$. MSCs are also easily obtained from other bone sources, such as humerus, femur, tibia, vertebral body or calcaneus and have their content ranges between $5.8 \times 10^{6}$ and $38.7 \times 10^{6}$ nucleated cells. Although, they present a wide range of documented nucleated cells, they can be cultivated and expanded in vitro in multiple cell types, avoiding a second surgical site while preventing post-operative pain and the possible risk for infection. Thus, they represent a promising and encouraging treatment option in orthopaedic surgery.
\end{abstract}

Keywords: Mesenchymal stem cells; iliac crest; femur; tibia; humerus

Received: 17 March 2020; Accepted: 31 July 2020; Published: 10 August 2020.

doi: $10.21037 /$ sci-2020-013

View this article at: http://dx.doi.org/10.21037/sci-2020-013

Nowadays, there is great hope worldwide addressed in regenerative medicine in all medical fields. Regenerative medicine has also opened a new horizon in orthopaedics, since modern regenerative procedures seem to offer an alternative treatment option for cartilage damage, osteoarthritis and large bone defects (1). The application of stem cells in regenerative medicine holds great potential and as a result, it is a particular appealing area of research in recent years.

Stem cells are undifferentiated cells characterized by two criteria: (I) a potential to self-renewal and (II) a potential to differentiate into multiple mature cell types. There are two broad categories of stem cells, embryonic and adult ones. Adult stem cells can be further subdivided into hematopoietic and mesenchymal stem cells (MSCs). MSCs are characterized as conductors of tissue repair and regeneration, by secreting trophic mediators (2). Therefore, MSCs are probably the most interesting for orthopaedic applications because of their potential to differentiate to both bone and cartilage $(1,2)$. MSCs can be isolated from various sources in the human body, such as bone marrow, adipose tissue, skin and skeletal muscle. Among these, bone marrow and adipose tissue derived MSCs are most commonly used, as they are easily obtained and abundantly available. Bone marrow derived MSCs (BM-MSCs) are currently considered the gold standard and the main source of MSCs for clinical applications in orthopaedics (3).

The challenge in orthopaedics focuses on repairing or regenerating damaged or diseased musculoskeletal tissues. MSCs are the most commonly used stem cells and are able to give rise to diverse tissues, including bone, cartilage, tendon, ligaments and muscle (2). These cells can be isolated from bone marrow and under controlled conditions; they can differentiate into fibroblast, chondrocyte, osteoblast and myoblast, making them useful for cell and tissue engineering for orthopaedic applications (3). 


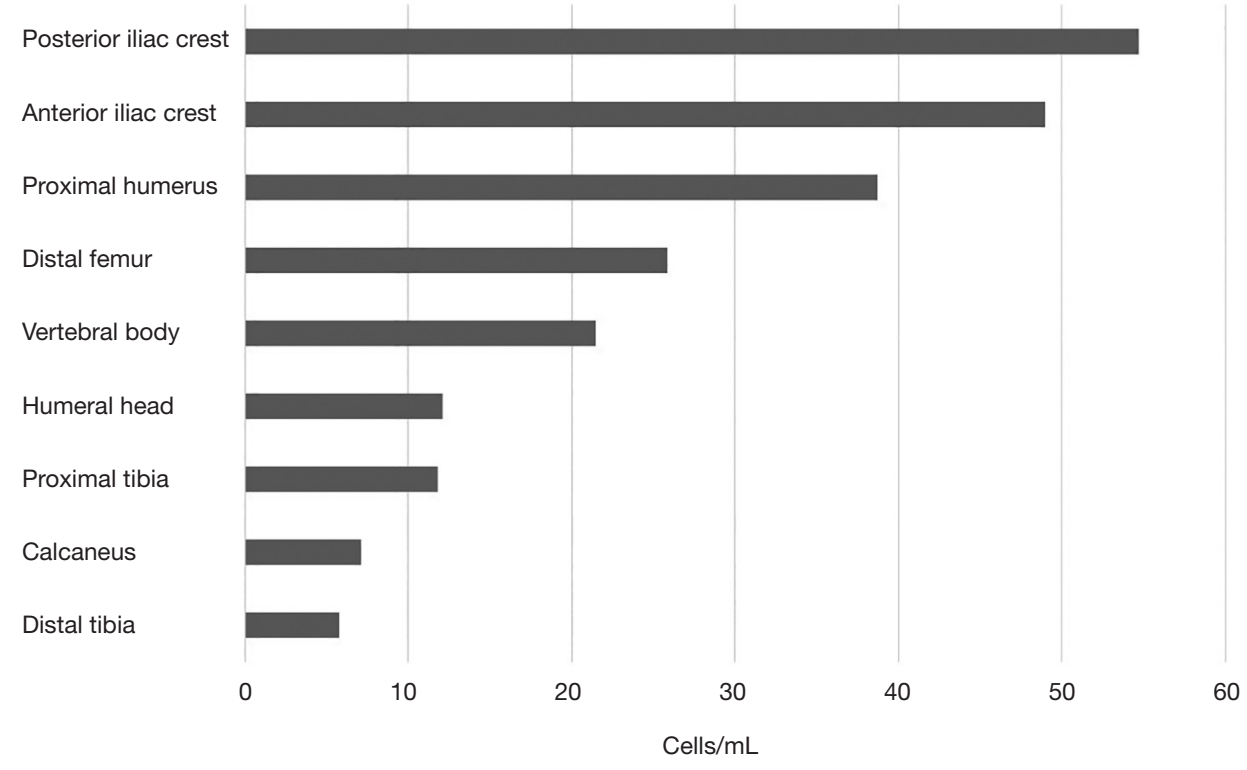

Figure 1 Comparison of nucleated cells derived from various bone sources.

Traditionally, the iliac crest represents the most common source of MSCs for clinical applications in orthopaedics (4-7). Recently, several reports have shown that these cells are also present in bone marrow from the proximal and distal femur $(4,7,8)$, proximal and distal tibia $(4,6,7)$, vertebral body (5), calcaneus (6), and humeral head (9). As for the iliac crest, a thorough literature search revealed that the ilium is the preferred donor site for obtaining autologous stem cells. The harvesting of bone marrow from the posterior iliac crest appears to be preferred, as it provided a modestly higher concentration of nucleated cells $\left[(25.1-54.7) \times 10^{6}\right.$ cells $\left./ \mathrm{mL}\right]$, while the anterior iliac crest had a mean concentration of $\left[(24.4-49) \times 10^{6}\right.$ cells $\left./ \mathrm{mL}\right](10,11)$ (Figure 1). Although, the differences are not statistically significant, it should be noted that the slight excess of posterior iliac crest could be relevant and may be due to aspiration techniques of each anatomic site.

Some studies have demonstrated other anatomical locations and have tried to identify the most appropriate location for sourcing MSCs. Beitzel et al. (12) compared bone marrow aspirates from the proximal humerus and distal femur during arthroscopic surgery. The mean number of $38.7 \times 10^{6}$ cells $/ \mathrm{mL}$ was reported from samples from the proximal humerus, while $25.9 \times 10^{6}$ cells $/ \mathrm{mL}$ for samples from the distal femur. Mazzocca et al. [2011] studied bone marrow of the humeral head from patients undergoing arthroscopic rotator cuff repair and found that the mean nucleated cells concentration was $12.1 \times 10^{6}$ cells $/ \mathrm{mL}$ (9), which is comparable to nucleated cells observed in the literature for the iliac crest and vertebral body $(5,6)$ (Figure 1).

Bone marrow of the vertebral body from patients undergoing posterior lumbar arthrodesis (5) and spinal fusion (13) demonstrated a mean concentration of $19.76 \times 10^{6}$ and $21.5 \times 10^{6}$ respectively (Figure 1 ). McLain et al. [2005] also studied bone marrow of the iliac crest and reported mean nucleated concentration of $16.95 \times 10^{6}(5)$. Thus, they suggested that there are equal numbers of nucleated cells in bone marrow harvested from the vertebral body and iliac crest $(5,6,13)$. As a result, harvesting bone marrow from the vertebral body can represent a promising alternative to the iliac crest, avoiding a second surgical site.

The tibial plateau, anterior ilium and posterior ilium were chosen as donor sites for patients who were undergoing bone regeneration for madibular or maxillary defects. The number of nucleated cells in bone marrow aspirates was $11.8 \times 10^{6}, 24.4 \times 10^{6}$, and $25.1 \times 10^{6}$ cells $/ \mathrm{mL}$, respectively (Figure 1). The yield of total nucleated cells was equal between the anterior and posterior ilium and double when compared to the tibial plateau (11). Regarding the ankle joint, Hyer et al. [2013] have recently published a study of bone marrow aspiration from distal tibial metaphysic and calcaneal body in 40 patients undergoing foot and ankle surgery. They demonstrated the presence of MSCs and their osteogenic potential. The number of 
nucleated cells in bone marrow aspirates was $5.8 \times 10^{6}$ and $7.1 \times 10^{6}$ cells $/ \mathrm{mL}$ for distal tibial metaphysic and calcaneal body, respectively (6) (Figure 1). According to the literature, the nucleated cells concentrations in iliac crest (10) were notable higher when compared with the proximal/ distal tibia and calcaneus (6). Patients undergoing ankle surgery were older, and as the number of proliferation rate of MSCs is thought to decrease with age, this may explain the significant lower nucleated cells concentrations reported here.

Direct comparison of amounts of isolated cells between different previously published studies may be difficult, since the differences in methods of isolation, culture times and differentiation media used, result in very diverse outcomes. Narbona-Carceles et al. [2011] (20 patients; mean age, 70.9 year; undergoing total knee arthroplasty) were able to isolate $(5.95 \pm 2.1) \times 10^{5} \mathrm{MSCs} / \mathrm{mL}$ from iliac crest samples, $(2.9 \pm 6.6) \times 10^{5} \mathrm{MSCs} / \mathrm{mL}$ from distal femur samples and $(3.25 \pm 2.6) \times 10^{5} \mathrm{MSCs} / \mathrm{mL}$ from proximal tibial samples (4). Juneja et al. [2010] (28 patients undergoing total hip arthroplasty and 10 patients undergoing total knee arthroplasty; age group 55-65 years) showed the viable MSCs in proximal and distal femur as $(4.1 \pm 0.72) \times 10^{6}$ and $(2.56 \pm 0.69) \times 10^{6}$, respectively (8). Hence, comparing the two studies for distal femur, there was no evidence of any major difference. Although, based on their data, Juneja et al. [2010] strongly recommended proximal femur as the preferred source for bone marrow collection, because of higher volume of blood that can be aspirated from this site, when compared to distal femur (8).

Modern strategies of tissue engineering and regenerative medicine utilize MSCs to improve bone repair and regeneration. They have been extensively studied because of their ability to differentiate into multiple cell types and their potential to increase osteoinduction and osteogenesis in bone repair $(1,10)$. Their outstanding capabilities rendered BM-MSCs widely applicable to regenerative medicine for the purpose of repairing and replacing damaged tissues. The major advantages of BM-MSCs are that they are an accessible source of cell harvesting, have high stability in culture, are ease of preparation and present high affinity to differentiate into osteoblastic lineage $(1,3)$.

Concluding, clinical studies have shown that BMMSCs can be harvest safely from different bone sources $(5,6,9,11)$. Originally, BM-MSCs were isolated from the iliac crest. This used to be the preferable source of MSCs for orthopaedic knee and shoulder surgeries $(4,10,11)$. However, this acquisition process is quite painful for the patient and also increases the risk of infection in bone tissue. In recent literature, various studies have described aspiration techniques (knee, shoulder and spine surgery) to harvest bone marrow during arthroscopic surgery and spine fusion without significant increases in operating time or patient complications and even re-injected to the repair site of the same patient to augment healing $(9,12,13)$. According to the International Society for Cellular Therapy, the minimum criteria for MSCs included plastic adherence, specific surface antigen expression and multipotent differentiation potential into adipocytes, osteoblasts and chondroblasts (14). Hopefully, the proximal humerus, distal femur and vertebral body aspiration yielded consistent volumes of bone marrow and nucleated cells comparable to aspirated from the iliac crest and were capable of undergoing differentiation into bone cells $(9,10,12)$.

\section{Acknowledgments}

Funding: None.

\section{Footnote}

Conflicts of Interest: Both authors have completed the ICMJE uniform disclosure form (available at http://dx.doi. org/10.21037/sci-2020-013). The authors have no conflicts of interest to declare.

Ethical Statement: The authors are accountable for all aspects of the work in ensuring that questions related to the accuracy or integrity of any part of the work are appropriately investigated and resolved.

Open Access Statement: This is an Open Access article distributed in accordance with the Creative Commons Attribution-NonCommercial-NoDerivs 4.0 International License (CC BY-NC-ND 4.0), which permits the noncommercial replication and distribution of the article with the strict proviso that no changes or edits are made and the original work is properly cited (including links to both the formal publication through the relevant DOI and the license). See: https://creativecommons.org/licenses/by-nc-nd/4.0/.

\section{References}

1. Paschos NK, Sennett ML. Update on mesenchymal stem cell therapies for cartilage disorders. World J Orthop 2017;8:853-60 
2. Kopka M, Bradley JP. The use of biologic agents in athletes with knee injuries. J Knee Surg 2016;29:379-86.

3. Nancarrow-Lei R, Mafi P, Mafi R, et al. A systematic review of adult mesenchymal stem cell sources and their multilineage differentiation potential relevant to musculoskeletal tissue repair and regeneration. Curr Stem Cell Res Ther 2017;12:601-10.

4. Narbona-Carceles J, Vaquero J, Suarez-Sancho S, et al. Bone marrow mesenchymal stem cell aspirates from alternative sources: Is the knee as good as the iliac crest? Injury 2014;45:S42-7.

5. McLain RF, Fleming JE, Boehm CA, et al. Aspiration of osteoprogenitor cells for augmenting spinal fusion: Comparison of progenitor cell concentrations from the vertebral bony and iliac crest. J Bone Joint Surg 2005;87:2655-61.

6. Hyer CE, Berlet GC, Bussewitz BW, et al. Quantitative assessment of the yield of osteoblastc connective tissue progenitors in bone marrow aspirate from the iliac crest, tibia, and calcaneus. J Bone Joint Surg Am 2013;95:1312-6.

7. Davies BM, Snelling SJB, Quek L, et al. Identifying the optimum source of mesenchymal stem cells for use in knee surgery. J Orthop Res 2017;35:1868-75.

8. Juneja SC, Viswanathan S, Ganguly M, et al. A simplified method for the aspiration of bone marrow from patients undergoing hip and knee replacement for isolating

doi: 10.21037/sci-2020-013

Cite this article as: Vasiliadis AV, Galanis N. Human bone marrow-derived mesenchymal stem cells from different bone sources: a panorama. Stem Cell Investig 2020;7:15. mesenchymal stem cells and in vitro chondrogenesis. Bone Marrow Res 2016;2016:3152065.

9. Mazzocca AD, McCarthy MBR, Chowaniec D, et al. Bone marrow-derived mesenchymal stem cells obtained during arthroscopic rotator cuff repair surgery show potential for tendon cell differentiation after treatment with insulin. Arthroscopy 2011;27:1459-71.

10. Pierini M, Di Bella C, Dozza B, et al. The posterior iliac crest outperforms the anterior iliac crest when obtaining mesenchymal stem cells from bone marrow. J Bone Joint Surg Am 2013;95:1101-7.

11. Marx RE, Tursun R. A qualitative and quantitative analysis of autologous human multipotent adult stem cells derived from three anatomic areas by marrow aspiration: Tibia, anterior ilium, and posterior ilium. Int J Oral Maxillofac Implants 2013;28:e290-4.

12. Beitzel K, McCarthy MBR, Cote MP, et al. Comparison of mesenchymal stem cells (osteoprogenitors) harvested from proximal humerus and distal femur during arthroscopic surgery. Arthroscopy 2013;29:301-8.

13. Hustedt JW, Jegede KA, Badrinath R, et al. Optimal aspiration volume of vertebral bone marrow for use in spinal fusion. Spine J 2013;13:1217-22.

14. Dominici M, Le Blanc K, Mueller I, et al. Minimal criteria for defining multipotent mesenchymal stromal cells. The International Society for Cellular Therapy position statement. Cytotherapy 2006;8:315-7. 